\title{
On the problem of axiomatization of tame representation type
}

\author{
by \\ Stanisław Kasjan (Toruń) \\ Dedicated to Professor Daniel Simson on the occasion \\ of his sixtieth birthday
}

\begin{abstract}
Associative algebras of fixed dimension over algebraically closed fields of fixed characteristic are considered. It is proved that the class of algebras of tame representation type is axiomatizable. Moreover, finite axiomatizability of this class is equivalent to the conjecture that the algebras of tame representation type form a Zariski-open subset in the variety of algebras.
\end{abstract}

1. Introduction. The aim of this paper is to discuss connections between model-theoretical properties of the class of algebras of tame representation type and the well known conjecture asserting that "tame representation type is open" (see [8]) in the sense explained below. Recall that a finite-dimensional algebra $R$ over an algebraically closed field is of tame representation type if in every dimension the indecomposable $R$-modules admit a parameterization by a finite number of one-parameter families. A precise formulation of this concept is given in Section 2.

Let us fix a natural number $d$ and a number $p$ which is zero or a prime. Consider the class $\mathbf{A l g}^{p}(d)$ of all associative algebras with identity having dimension $d$ over the base field, which is assumed to be algebraically closed of characteristic $p$. This class is axiomatizable in a suitable first order language. Let $\mathcal{T}$ be the subclass consisting of algebras of tame representation type.

We prove in Theorem 2.3 that $\mathcal{T}$ is axiomatizable and an explicit system of axioms is presented in Proposition 3.3. Our main result is Theorem 2.4 asserting that finite axiomatizability of this class is equivalent to the fact that "tame is open", that is, the tame algebras induce an open (in the Zariski topology) subset in the variety of algebras over each algebraically

2000 Mathematics Subject Classification: 16G60, 03C60, 03 C98.

Supported by Polish KBN Grant 2 P03A 01216. 
closed field (of characteristic $p$ ). According to the author's knowledge, the question whether $\mathcal{T}$ is indeed finitely axiomatizable is still open.

The analogous problem for finite representation type was solved by Jensen and Lenzing (see [11, Theorem 12.54], [10]). They prove that the class of all algebras of finite representation type having a fixed dimension over the (algebraically closed) base field, as well as the class of algebras of infinite representation type, are finitely axiomatizable.

This is related to the theorem of Gabriel [6] that "finite representation type is open". One can observe that the final part of Gabriel's proof can be expressed very naturally in model-theoretical terms using the results of Jensen and Lenzing.

The paper is organized as follows. Section 2 contains some preliminary material and the formulation of the main theorems. In Section 3 we present a set of axioms for the class of tame algebras and prove in 3.6 that finite axiomatizability of this class implies openness of the set of tame algebras in the variety of algebras. A result of Geiss [8] asserting that a degeneration of a wild algebra is wild, or rather his main arguments for that theorem, are an important ingredient of our proof.

The converse of the implication proved in 3.6 is shown (for a fixed characteristic) in Section 4, where also a useful "geometric" criterion for axiomatizability is given. In Section 5 we restrict our attention to the class $\mathcal{Q}$ of quasitilted algebras. We show in Lemma 5.1 that the tame quasitilted algebras form a finitely axiomatizable subclass of $\mathcal{Q}$ and we discuss some consequences of our main results.

2. Preliminaries. Let $\mathbb{L}$ be the first order language having countably many variables, two binary function symbols + and $\cdot$ and two constants 0,1 . We think about $\mathbb{L}$ as a language of the first order theory of fields or rings with identity. Denote by $\mathbb{A}$ the two-sorted first order language of algebras over fields (see [11]), that is, the disjoint union $\mathbb{L}_{1} \amalg \mathbb{L}_{2}$ of two copies of $\mathbb{L}$ equipped with another function symbol $\cdot$. The terms from $\mathbb{L}_{1}$ (resp. $\mathbb{L}_{2}$ ) are called terms of the first (resp. second) sort. The new function symbol associates to a pair of variables of first and second sort a variable of the second sort. The language $\mathbb{A}$ has the usual logical connectives: $\wedge, \vee, \neg, \rightarrow$ and allows quantifiers on both sorts of variables.

By a model for this language we mean a pair $(K, R)$, where $K$ and $R$ are models for $\mathbb{L}_{1}$ and $\mathbb{L}_{2}$ respectively and the new function symbol is interpreted as a function

$$
\cdot: K \times R \rightarrow R
$$

It is clear that if $K$ is a field and $R$ is a $K$-algebra with identity then the obvious interpretation of the symbols of the language $\mathbb{A}$ allows us to treat the pair $(K, R)$ as a model for $\mathbb{A}$. 
Fix a natural number $d$ and let $\operatorname{Alg}(d)$ be the class of all models $(K, R)$ for $\mathbb{A}$ such that $K$ is an algebraically closed field and $R$ is a $d$-dimensional associative $K$-algebra with identity. If $p$ is zero or a prime we denote by $\mathbf{A l g}^{p}(d)$ the class of all models $(K, R)$ in $\mathbf{A} \lg (d)$ such that the characteristic of the field $K$ is $p$. It is easy to observe that $\mathbf{A} \lg (d)$ and $\mathbf{A l g}^{p}(d)$ are (nonfinitely!) axiomatizable classes of models.

We use the standard notation: for a model $(K, R)$ and a first order formula $\phi$ we write $(K, R) \models \phi$ if $\phi$ is satisfied in $(K, R)$. Two models are elementarily equivalent if the sets of formulas satisfied in each of them coincide.

A nonempty family $\mathcal{F}$ of subsets of a set $I$ is called an ultrafilter over $I$ if the following conditions are satisfied:

- $\emptyset \notin \mathcal{F}$,

- if $A, B \in \mathcal{F}$ then $A \cap B \in \mathcal{F}$,

- if $A \in \mathcal{F}$ and $B \subseteq I$ then $A \cup B \in \mathcal{F}$,

- if $A \notin \mathcal{F}$ then $I \backslash A \in \mathcal{F}$.

Given a family $\left(M_{i}\right)_{i \in I}$ of sets the ultrafilter $\mathcal{F}$ over $I$ induces an equivalence relation $\sim$ in the product $\prod_{i \in I} M_{i}$ defined by $\left(m_{i}\right)_{i \in I} \sim\left(m_{i}^{\prime}\right)_{i \in I}$ if and only if $m_{i}=m_{i}^{\prime}$ for $\mathcal{F}$-almost all $i \in I$, that is, there is $U \in \mathcal{F}$ such that $m_{i}=m_{i}^{\prime}$ for all $i \in U$. We denote by $\left(m_{i}\right)^{\mathcal{F}}$ the equivalence class of an element $\left(m_{i}\right)$ with respect to the relation $\sim$ and by $\prod M_{i} / \mathcal{F}$ the ultraproduct of $\left(M_{i}\right)_{i \in I}$ with respect to $\mathcal{F}$, that is, the set of all equivalence classes of $\sim$. To simplify the formulas we omit the condition $i \in I$ in the notation of the ultraproduct; it does not lead to confusion.

If $\left(K_{i}, R_{i}\right)_{i \in I}$ is a family of models for $\mathbb{A}$ then by its ultraproduct we mean the model

$$
\prod\left(K_{i}, R_{i}\right) / \mathcal{F}=\left(\prod K_{i} / \mathcal{F}, \prod R_{i} / \mathcal{F}\right)
$$

with the obvious interpretations of all symbols in $\mathbb{A}$.

The concept of ultraproduct appears in [14] under the name "operation (P)". The importance of this construction is established by the famous Łoś Ultraproduct Theorem asserting that a first order sentence $\phi$ is satisfied in $\prod\left(K_{i}, R_{i}\right) / \mathcal{F}$ if and only if it is satisfied in $\left(K_{i}, R_{i}\right)$ for $\mathcal{F}$-almost all $i \in I$ (see [11, Theorem 1.5], [2, Theorem 4.1.9]).

Given a model $(K, R)$ its ultrapower with respect to $\mathcal{F}$ is defined to be the model $(K, R)^{I} / \mathcal{F}=\prod\left(K_{i}, R_{i}\right) / \mathcal{F}$, where $\left(K_{i}, R_{i}\right)=(K, R)$ for every $i \in I$.

A class $\mathcal{C}$ of models is axiomatizable if there is a set $\Sigma$ of first order sentences such that a model $(K, R)$ belongs to $\mathcal{C}$ if and only if all sentences of $\Sigma$ are satisfied in $(K, R)$. A class $\mathcal{C}$ is axiomatizable if and only if it is closed under elementary equivalence and formation of ultraproducts $[14$, 2.6], [11, Theorem 2.12]. 
Let $\mathcal{C} \subseteq \mathcal{B}$ be classes of models. A set $\Sigma$ of sentences is a set of axioms for $\mathcal{C}$ as a subclass of $\mathcal{B}$ if for every model $M$ in $\mathcal{B}: M$ belongs to $\mathcal{C}$ if and only if all sentences of $\Sigma$ are satisfied in $M$. The class $\mathcal{C}$ is finitely axiomatizable as a subclass of $\mathcal{B}$ provided there exists a finite set of axioms for $\mathcal{C}$ as a subclass of $\mathcal{B}$. This is equivalent to the existence of a set of axioms containing one element.

The following theorem follows by standard model-theoretical arguments [11, Theorem 2.13], [2, Theorem 4.1.12].

Theorem 2.1. Let $\mathcal{B}$ be an axiomatizable class of models. $A$ class $\mathcal{C} \subseteq \mathcal{B}$ is finitely axiomatizable as a subclass of $\mathcal{B}$ if and only if both classes $\mathcal{C}$ and $\mathcal{B} \backslash \mathcal{C}$ are axiomatizable. If this is the case then from every set of axioms for $\mathcal{C}$ as a subclass of $\mathcal{B}$ one can choose a finite one.

The reader is referred to [2], [11], [15] for detailed expositions of model theory.

With a field $K$ and a system $\gamma=\left(\gamma_{i j k}\right)_{i, j, k=1}^{d} \in K^{d^{3}}$ we associate a model $\left(K, R_{K}(\gamma)\right)$ for $\mathbb{A}$ such that $R_{K}(\gamma)=K^{d}$ as a vector space and the multiplication - in $R_{K}(\gamma)$ is given by the structure constants $\gamma$, that is,

$$
e_{i} \cdot e_{j}=\sum_{k=1}^{d} \gamma_{i j k} e_{k}
$$

for all $i, j, 1 \leq i, j \leq d$, if we denote the $i$ th standard basis vector of $R_{K}(\gamma)$ by $e_{i}$. It is known (see [6], [13]) that the tuples $\gamma$ such that $\left(K, R_{K}(\gamma)\right) \in$ $\operatorname{Alg}(d)$ form an affine algebraic variety $\operatorname{Alg}_{K}(d)$ for every algebraically closed field $K$.

Given a field $K$ and a class $\mathcal{C} \subseteq \operatorname{Alg}(d)$ we denote by $\mathcal{C}_{K}$ the preimage of $\mathcal{C}$ under the map $\gamma \mapsto\left(K, R_{K}(\gamma)\right)$, that is,

$$
\mathcal{C}_{K}=\left\{\gamma \in K^{d^{3}}:\left(K, R_{K}(\gamma)\right) \in \mathcal{C}\right\} .
$$

Recall that a finite-dimensional algebra $R$ over an algebraically closed field $K$ is of tame representation type provided for every number $m$ there exists a finite family $N_{1}, \ldots, N_{r}$ of $K[t]-R$-bimodules that are free of rank $m$ as left $K[t]$-modules and such that every indecomposable $m$-dimensional $R$-module is isomorphic to $K[t] /(t-\lambda) \otimes_{K[t]} N_{i}$ for suitable $i \leq r$ and $\lambda \in K$ (see [19, Chapter 14]).

The algebra $R$ is of wild representation type if there exists a $K\langle X, Y\rangle$ $R$-bimodule $M$ free of finite rank over $K\langle X, Y\rangle$ such that the functor

$$
(-) \otimes_{K\langle X, Y\rangle} M: \operatorname{fin}(K\langle X, Y\rangle) \rightarrow \bmod (R)
$$

preserves indecomposability and sends nonisomorphic modules to nonisomorphis ones. Here $K\langle X, Y\rangle$ is the free associative $K$-algebra with two free 
generators $X$ and $Y$ and $\operatorname{fin}(K\langle X, Y\rangle)$ is the category of finite-dimensional right $K\langle X, Y\rangle$-modules [4], [5].

We fix $p$ which is zero or a prime and from now on consider algebraically closed fields of characteristic $p$. Let $\mathcal{T}$ (resp. $\mathcal{W}$ ) denote the class of all models $(K, R) \in \mathbf{A l g}^{p}(d)$ such that $R$ is of tame (resp. wild) representation type. The famous Tame-Wild Dichotomy of Drozd [5], [3] can be expressed in the following form.

Theorem 2.2. The class $\mathbf{A l g}^{p}(d)$ is the disjoint union of $\mathcal{T}$ and $\mathcal{W}$.

The main results of this paper are the following two theorems.

TheOREM 2.3. The class $\mathcal{T}$ of tame algebras is axiomatizable.

THEOREM 2.4. The following assertions are equivalent:

(A) For every algebraically closed field $K$ of characteristic $p$ the set $\mathcal{W}_{K}$ is Zariski-closed in $\mathbf{A l g}_{K}(d)$.

(B) There exists polynomials $H_{1}, \ldots, H_{r}$ in $d^{3}$ variables with coefficients in the prime field $\mathbb{F}_{p}$ of characteristic $p$ such that

$$
\mathcal{W}_{K}=\left\{\gamma \in \operatorname{Alg}_{K}(d): H_{1}(\gamma)=\ldots=H_{r}(\gamma)=0\right\}
$$

for every algebraically closed field $K$ of characteristic $p$.

(C) The class $\mathcal{T}$ is finitely axiomatizable as a subclass of $\mathbf{A l g}^{p}(d)$.

(D) The class $\mathcal{W}$ is axiomatizable.

(E) The class $\mathcal{W}$ is finitely axiomatizable as a subclass of $\operatorname{Alg}^{p}(d)$.

The proofs of Theorems 2.3 and 2.4 are given in Section 3 .

REMARK 2.4. It follows easily from the proof which we present below that fixing the characteristic $p$ is not essential for the implication $(\mathrm{C}) \Rightarrow(\mathrm{A})$ and for the equivalence of (C), (D) and (E). So the following two assertions are equivalent:

1. the tame algebras (of fixed dimension $d$ ) form a finitely axiomatizable class,

2. the wild algebras form an axiomatizable class.

Both assertions imply that the tame algebras induce an open subset of the variety of $d$-dimensional algebras for every algebraically closed field.

3. A set of axioms for tame algebras. In this section we give a set of axioms for the class of tame algebras. Let $(K, R) \in \mathbf{A l g}^{p}(d)$. Fix a basis in $R$ and observe that any $K\langle X, Y\rangle$-R-bimodule $M$ free of rank $m$ over $K\langle X, Y\rangle$ can be represented by a sequence $M_{1}, \ldots, M_{d}$ of $m \times m$ matrices with coefficients in $K\langle X, Y\rangle$. By the degree of $M$ we mean the minimal upper bound for the degrees of these coefficients, where the minimum is taken over all possible presentations $M_{1}, \ldots, M_{d}$. 
Fix two numbers $m$ and $l$. Observe that the following property of $(K, R)$ can be expressed in the language $\mathbb{A}$ :

"There exists a $K\langle X, Y\rangle$-R-bimodule $M$ free of rank $m$ and of degree at most $l$ such that for any $m$-dimensional right $K\langle X, Y\rangle$-modules $U, V$ the $R$-modules $U \otimes_{K\langle X, Y\rangle} M$ and $V \otimes_{K\langle X, Y\rangle} M$ are isomorphic if and only if $U \cong V$."

Denote the corresponding sentence by $\omega_{m, l}$. Given a field $K$ let

$$
W_{K}(m, l)=\left\{\gamma \in \operatorname{Alg}_{K}(d):\left(K, R_{K}(\gamma)\right)=\omega_{m, l}\right\} .
$$

Lemma 3.1. (a) Let $(K, R) \in \operatorname{Alg}^{p}(d)$. Then $(K, R) \in \mathcal{W}$ if and only if $(K, R) \mid=\omega_{m, l}$ for some $m, l$.

(b) $\overline{W_{K}(m, l)} \subseteq \mathcal{W}_{K}$ for every algebraically closed field $K$.

Proof. The implication " $\Rightarrow$ " in (a) is a direct consequence of the definition of wild representation type, whereas the opposite one follows from (b). The assertion (b) is implicitly proved by Geiss in [8, Proposition 2]. For convenience of the reader we recall the main arguments (see also [16]).

For this we need some notation introduced in [8]. Given a number $z$ denote by $\bmod _{R}(z)$ the variety of $R$-module structures on $K^{z}$ and for any $t \leq z^{2}$ define $\bmod _{R}(z, t)$ to be the (closed) subset of $\bmod _{R}(z)$ consisting of those module structures whose endomorphism rings have dimension at least $t$.

The following facts are established in [8]:

1. $R$ is tame if and only if $\operatorname{dim} \bmod _{R}(z, t) \leq z^{2}+z-t$ for every $z \in \mathbb{N}$ and $t \leq z^{2}$, and

2. the function

$$
\Theta_{z, t}: \operatorname{Alg}_{K}(d) \rightarrow \mathbb{N}, \quad \gamma \mapsto \operatorname{dim} \bmod _{R(\gamma)}(z, t),
$$

is upper semicontinuous for every $z \in \mathbb{N}$ and $t \leq z^{2}$.

Now assume that $(K, R) \models \omega_{m, l}$. The bimodule $M$ whose existence is postulated by the sentence $\omega_{m, l}$ induces a regular map

$$
\ell: \mathcal{U} \rightarrow \bmod _{R}\left(m^{2}\right),
$$

where $\mathcal{U}=\mathbb{M}_{m}(K) \times \mathbb{M}_{m}(K)$. Here $\mathbb{M}_{m}(K)$ denotes the variety of all $m$ by $m$ matrices with coefficients in $K$.

Recall that the general linear group $\mathbf{G l}_{m^{2}}(K)$ acts on $\bmod { }_{R}\left(m^{2}\right)$ by conjugation in such a way that the orbits of this action correspond to the isomorphism classes of $m^{2}$-dimensional $R$-modules. Consider the map

$$
\varrho: \mathbf{G l}_{m^{2}}(K) \times \mathcal{U} \rightarrow \bmod _{R}\left(m^{2}\right)
$$

given by $\varrho(g, a, b)=g \ell(a, b) g^{-1}$. Let $x$ be an element of the image of $\varrho$ having the minimal dimension of the endomorphism ring, say, $t_{0}$. Then the image of 
$\varrho$ is contained in $\bmod _{R}\left(m^{2}, t_{0}\right)$. Let us investigate the fibre $\varrho^{-1}(x)$. Assume that $\varrho(g, a, b)=\varrho\left(g_{1}, a_{1}, b_{1}\right)=x$. By properties of the bimodule $M$ described by $\omega_{m, l}$ there exists $h \in \mathbf{G l}_{m}(K)$ such that $\left(h a h^{-1}, h b h^{-1}\right)=\left(a_{1}, b_{1}\right)$. The matrix $h$ induces (in a regular way) an element $\widetilde{h} \in \mathbf{G l}_{m^{2}}(K)$ such that $\widetilde{h} \ell(a, b) \widetilde{h}^{-1}=\ell\left(a_{1}, b_{1}\right)$. Then the element $g_{1} \widetilde{h} g^{-1}$ belongs to the stabilizer $\operatorname{Stab}(x)$ of $x$. Now it is immediate that the image of the regular map

$$
u: \mathbf{S t a b}(x) \times \mathbf{G l}_{m}(K) \rightarrow \mathbf{G l}_{m^{2}}(K) \times \mathcal{U}
$$

defined by $(s, h) \mapsto\left(s g \widetilde{h}^{-1}, h a h^{-1}, h b h^{-1}\right)$ equals $\varrho^{-1}(x)$. Each fibre of $u$ is at least one-dimensional, hence

$$
\operatorname{dim} \varrho^{-1}(x) \leq t_{0}+m^{2}-1
$$

by the Fibre Dimension Theorem [18, Chapter I.6] and the equality $\operatorname{dim} \operatorname{Stab}(x)=t_{0}$.

Applying that theorem again to the map $\varrho$ (note that $\mathbf{G l}_{m^{2}}(K) \times \mathcal{U}$ is irreducible) we get

$\operatorname{dim} \bmod _{R}\left(m^{2}, t_{0}\right) \geq \operatorname{dim}\left(\mathbf{G l}_{m^{2}}(K) \times \mathcal{U}\right)-\left(t_{0}+m^{2}-1\right)=m^{4}+m^{2}-t_{0}+1$.

We have shown that

$$
W_{K}(m, l) \subseteq \bigcup_{t \leq m^{4}}\left\{\gamma \in \operatorname{Alg}_{K}(d): \Theta_{m^{2}, t}(\gamma)>m^{4}+m^{2}-t_{0}\right\} .
$$

Since, by observations 1 and 2 above, the right hand side is a Zariski-closed set contained in $\mathcal{W}_{K}$, the proof is complete.

Thanks to Lemma 3.1(b) we have the following generalization of the theorem on degenerations of algebras [8]:

Corollary 3.2. Let $\gamma \in \mathcal{W}_{K}$. Denote by $\operatorname{El}(\gamma)$ the set of all points $\gamma^{\prime} \in \mathbf{A l g}_{K}(d)$ such that the algebras $\left(K, R_{K}(\gamma)\right)$ and $\left(K, R_{K}\left(\gamma^{\prime}\right)\right)$ are elementarily equivalent. Then

$$
\overline{\operatorname{El}(\gamma)} \subseteq \mathcal{W}_{K}
$$

Theorem 2.3 is a direct consequence of the following proposition.

Proposition 3.3. The set

$$
\Omega=\left\{\neg \omega_{m, l}: m, l \in \mathbb{N}\right\}
$$

is a set of axioms for the class $\mathcal{T}$ of tame algebras.

Proof. Apply Lemma 3.1(a).

Corollary 3.4. (a) The class $\mathcal{T}$ is closed under elementary equivalence and the formation of ultraproducts.

(b) The class $\mathcal{W}$ is closed under elementary equivalence.

Remark 3.5. The system of axioms in Proposition 3.3 is not optimal. It is arranged in this way to have the geometric property in Lemma 3.1(b). 
Perhaps more natural is the system of sentences asserting the existence of bimodules $M$ satisfying the condition: $U \otimes M \cong V \otimes M \Rightarrow U \cong V$ for one-dimensional modules $U, V$ instead of $m$-dimensional. Moreover by the results of [7] it is enough to restrict the attention to bimodules of degree 1.

The crucial problem is to decide whether our system of axioms can be replaced by a finite one.

Now we are in a position to prove one of the implications in Theorem 2.4 .

3.6. Proof of $(\mathrm{C}) \Rightarrow(\mathrm{A})$. Assume that $\mathcal{T}$ is finitely axiomatizable as a subclass of $\mathbf{A l g}^{p}(d)$. It follows (see Theorem 2.1) that there exists a finite subset of the set $\Omega$ which is already a system of axioms for $\mathcal{T}$ as a subclass of $\mathbf{A l g}^{p}(d)$. Let $N$ be a number such that for every $(K, R) \in \mathbf{A l g}^{p}(d)$ the model $(K, R)$ is in $\mathcal{W}$ if and only if $(K, R) \models \omega_{m, l}$ for some $m, l \leq N$. In terms of the corresponding subsets of the algebra varieties this means that

$$
\mathcal{W}_{K}=\bigcup_{m, l=1}^{N} W_{K}(m, l)
$$

for every field $K$. But $\overline{W_{K}(m, l)} \subseteq \mathcal{W}_{K}$, by Lemma 3.1(b). Hence

$$
\mathcal{W}_{K}=\bigcup_{m, l=1}^{N} \overline{W_{K}(m, l)}
$$

and $\mathcal{W}_{K}$ is closed in $\operatorname{Alg}_{K}(d)$.

Corollary 3.7. Assume that assertion (C) is true. Then there is a number $N$ such that for any algebraically closed field $K$ of characteristic $p$ and any $d$-dimensional $K$-algebra $R$ of wild representation type there exists a $K\langle X, Y\rangle$-R-bimodule $M$ free of rank $m \leq N$ such that for any $m$ dimensional right $K\langle X, Y\rangle$-modules $U, V$ the $R$-modules $U \otimes_{K\langle X, Y\rangle} M$ and $V \otimes_{K\langle X, Y\rangle} M$ are isomorphic if and only if $U \cong V$.

The corollary is an immediate consequence of the above proof. Varying the system of axioms for $\mathcal{T}$ we obtain corresponding variations of the corollary. However it is not clear if under assumption (C) one can prove that there is a number $N$ such that whenever $(K, R) \in \mathcal{W}$ then there exists a $K\langle X, Y\rangle$-R-bimodule $M$ free of $K\langle X, Y\rangle$-rank less than or equal to $N$ such that the functor

$$
(-) \otimes_{K\langle X, Y\rangle} M: \operatorname{fin}(K\langle X, Y\rangle) \rightarrow \bmod (R)
$$

preserves indecomposability and sends nonisomorphic modules to nonisomorphic ones. 
4. A sufficient condition for axiomatizability. In order to prove the remaining part of Theorem 2.4 we work in a little more general context. Throughout this section $\mathcal{C}$ is a subclass of $\mathbf{A} \lg ^{p}(d)$. We say that $\mathcal{C}$ is geometrically closed provided for every algebraically closed field of characteristic $p$ the set $\mathcal{C}_{K}$ is a closed subset of $\operatorname{Alg}_{K}(d)$.

Proposition 4.1. If $\mathcal{C}$ is closed under elementary equivalence and geometrically closed then it is closed under formation of arbitrary ultraproducts and hence axiomatizable.

Before giving the proof of the proposition we show that $\mathcal{C}$ is closed under base field extensions. Given a $K$-algebra $R$ and a field extension $K \subseteq L$ we set $R^{(L)}=R \otimes_{K} L$ and view it as an $L$-algebra in the obvious way.

LEMMA 4.2. Assume that $\mathcal{C}$ is closed under elementary equivalence, $K \subseteq L$ is a field extension of algebraically closed fields of characteristic $p$ and $(K, R) \in \mathbf{A l g}^{p}(d)$. Then $(K, R) \in \mathcal{C}$ if and only if $\left(L, R^{(L)}\right) \in \mathcal{C}$.

Proof. Let $I$ be a set of cardinality greater than or equal to the cardinality of $L$ and let $\mathcal{F}$ be a regular ultrafilter over $I$ [15, Definition 18.34]. Then by [15, Theorem 18.37] the ultrapowers $K^{I} / \mathcal{F}$ and $L^{I} / \mathcal{F}$ are algebraically closed fields of characteristic $p$ with the same cardinality $2^{|I|}>$ $\max \{|L|,|K|\}$. There are canonical diagonal embeddings

$$
\Delta: K \rightarrow K^{I} / \mathcal{F}, \quad \Delta: L \rightarrow L^{I} / \mathcal{F}
$$

and we treat the ultrapowers as extensions of $K$. It follows that they have the same transcendence degree over $K$ and hence they are $K$-isomorphic, that is, there exists a field isomorphism $\sigma$ making the diagram

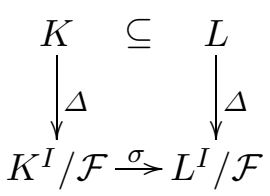

commutative.

The isomorphism $\sigma$ induces an isomorphism of models

$$
(K, R)^{I} / \mathcal{F} \cong\left(L, R^{(L)}\right)^{I} / \mathcal{F} .
$$

Thanks to the Eoś Ultraproduct Theorem [11, Theorem 1.5], [2, Theorem 4.1.9] the model $(K, R)$ is elementarily equivalent to the left hand side model whereas $\left(L, R^{(L)}\right)$ is elementarily equivalent to the other one. Thus our assertion follows from the assumption that $\mathcal{C}$ is closed under elementary equivalence.

Applying Lemma 4.2 to $\mathcal{C}=\mathcal{W}$ we see that $R$ is of tame representation type if and only if so is $R^{(L)}$ (comp. [12]). 
Assume that $L$ is the ultrapower $K^{I} / \mathcal{F}$ of a field $K$. For any number $n$ we denote by $\Delta: K^{n} \rightarrow L^{n}$ the map induced by the diagonal embedding. Moreover if $x^{i}=\left(x_{1}^{i}, \ldots, x_{n}^{i}\right) \in K^{n}$ for $i \in I$ then we denote the element $\left(\left(x_{1}^{i}\right)^{\mathcal{F}}, \ldots,\left(x_{n}^{i}\right)^{\mathcal{F}}\right)$ of $L^{n}$ by $\left(x^{i}\right)^{\mathcal{F}}$.

Lemma 4.3. Assume that the set $V \subseteq L^{n}$ is Zariski-closed (over $L$ ) and there are points $x^{i} \in K^{n}$ such that $\Delta\left(x^{i}\right) \in V$ for $\mathcal{F}$-almost all $i \in I$. Then

$$
\left(x^{i}\right)^{\mathcal{F}} \in V .
$$

Proof. Without loss of generality we can assume that $\Delta\left(x^{i}\right) \in V$ for every $i \in I$. Let $H \in L\left[X_{1}, \ldots, X_{n}\right]$ be a polynomial vanishing on $V$; assume that it has degree $r$. There exist polynomials $H^{i} \in K\left[X_{1}, \ldots, X_{n}\right], i \in I$, of degree at most $r$ such that $H=\left(H^{i}\right)^{\mathcal{F}}$. The condition $H\left(\Delta\left(x^{i}\right)\right)=0$ means that there exists a set $U_{i} \in \mathcal{F}$ such that $H^{j}\left(x^{i}\right)=0$ for $j \in U_{i}$.

Let $T$ be the space of $K$-linear functionals on the (finite-dimensional) space of polynomials in $K\left[X_{1}, \ldots, X_{n}\right]$ of degree at most $r$ and let $\xi^{i} \in T$ be the evaluation at $x^{i}$ for $i \in I$. Let $\xi^{i_{1}}, \ldots, \xi^{i_{s}}$ be a basis of the subspace generated by all $\xi^{i}, i \in I$. Then

$$
\xi^{i}\left(H^{j}\right)=H^{j}\left(x^{i}\right)=0
$$

for $j \in U_{i_{1}} \cap \ldots \cap U_{i_{s}}$ and arbitrary $i \in I$. In particular $H^{j}\left(x^{j}\right)=0$. Since $U_{i_{1}} \cap \ldots \cap U_{i_{s}} \in \mathcal{F}$ this means that

$$
H\left(\left(x^{i}\right)^{\mathcal{F}}\right)=\left(H^{i}\left(x^{i}\right)\right)^{\mathcal{F}}=0
$$

and we are done.

Proof of Proposition 4.1. Take a family $\left(K_{i}, R_{i}\right), i \in I$, of models in $\mathcal{C}$ and an arbitrary ultrafilter $\mathcal{F}$ over $I$. It is sufficient to prove that the ultraproduct $\prod\left(K_{i}, R_{i}\right) / \mathcal{F}$ belongs to $\mathcal{C}$.

Since all fields $K_{i}$ have the same characteristic $p$ there exists an algebraically closed field $K$ and field embeddings $K_{i} \subseteq K$ for $i \in I$. Observe that there is an isomorphism of models

$$
\prod\left(K, R_{i} \otimes_{K_{i}} K\right) / \mathcal{F} \cong\left(L,\left(\prod R_{i} / \mathcal{F}\right) \otimes_{\left(\prod K_{i} / \mathcal{F}\right)} L\right)
$$

where $L$ is the ultrapower $K^{I} / \mathcal{F}$.

Thus thanks to Lemma 4.2 it is enough to prove the assertion under the assumption that $K_{i}=K$ for $i \in I$.

Let $\gamma^{i} \in \mathbf{A l g}_{K}(d)$ be the point corresponding to the algebra $R_{i}$. Then $\Delta\left(\gamma^{i}\right)$ corresponds to the $L$-algebra $R_{i}^{(L)}$ and $\left(\gamma^{i}\right)^{\mathcal{F}}$ corresponds to $\prod R_{i} / \mathcal{F}$. By our assumptions and Lemma 4.2 we know that $\Delta\left(\gamma^{i}\right) \in \mathcal{C}_{L}$ for $i \in I$. Since $\mathcal{C}_{L}$ is Zariski-closed we conclude that $\left(\gamma^{i}\right)^{\mathcal{F}} \in \mathcal{C}_{L}$ by Lemma 4.3. But this means that $\left(L, \prod R_{i} / \mathcal{F}\right) \in \mathcal{C}$ as required. 
Proposition 4.4. Assume that $\mathcal{C}$ is finitely axiomatizable as a subclass of $\mathbf{A l g}^{p}(d)$ and geometrically closed. If $\mathbb{F}_{p}$ is the prime field of characteristic $p$ then there exist polynomials

$$
H_{1}, \ldots, H_{r} \in \mathbb{F}_{p}\left[X_{i j k}\right]_{i, j, k=1, \ldots, d}
$$

such that

$$
\mathcal{C}_{K}=\left\{\gamma \in \operatorname{Alg}_{K}(d): H_{1}(\gamma)=\ldots=H_{r}(\gamma)=0\right\}
$$

for every algebraically closed field $K$ of characteristic $p$.

Proof. Denote by $M$ the algebraic closure of $\mathbb{F}_{p}$. Let $F_{1}, \ldots, F_{s}$ be polynomials defining the closed set $\mathcal{C}_{M}$ in $\operatorname{Alg}_{K}(d)$. There is a finite Galois extension $\mathbb{F}_{p} \subseteq N$ such that all coefficients of all polynomials $F_{1}, \ldots, F_{s}$ belong to $N$. Since the class $\mathcal{C}$ is closed under elementary equivalence it follows that $\mathcal{C}_{M}$ is stable under the action of the Galois group $G=G\left(N / \mathbb{F}_{p}\right)$ of the extension $\mathbb{F}_{p} \subseteq N$.

Let $G=\left\{\sigma_{1}, \ldots, \sigma_{t}\right\}$, where $t=\left[N: \mathbb{F}_{p}\right]$. It follows that the polynomial $F_{i}^{\sigma_{j}}$ (the result of the action of $\sigma_{j}$ on $F_{i}$ ) vanishes on $\mathcal{C}_{M}$ for every $i=1, \ldots, s, j=1, \ldots, t$. Let $S_{1}, \ldots, S_{t}$ be the elementary symmetric polynomials in $t$ variables. It is easy to observe that the polynomials

$$
S_{k}\left(F_{i}^{\sigma_{1}}, \ldots, F_{i}^{\sigma_{t}}\right), \quad i=1, \ldots, s, k=1, \ldots, t,
$$

have coefficients in $\mathbb{F}_{p}$ and define the variety $\mathcal{C}_{M}$.

Denote these polynomials by $H_{1}, \ldots, H_{r}$, where $r=s t$.

Now let $\phi$ be the axiom for $\mathcal{C}$ as a subclass of $\operatorname{Alg}_{K}(d)$. Denote by $\psi_{\phi}(\gamma)$ the corresponding formula in the language $\mathbb{L}$ expressing $\phi$ in terms of the structure constants. More precisely, for $\gamma \in \mathbf{A l g}_{K}(d)$ the formula $\psi_{\phi}(\gamma)$ means that $\left(K, R_{K}(\gamma)\right) \models \phi$ (see the proof of Corollary 12.57 in [11]). Let $\alpha(\gamma)$ be a formula meaning that $\left(L, R_{L}(\gamma)\right) \in \mathbf{A l g}^{p}(d)$ for $\gamma \in L^{d^{3}}$ and every algebraically closed field $L$ of characteristic $p$.

We conclude that

$$
M \models\left[\alpha(\gamma) \rightarrow\left(\psi_{\phi}(\gamma) \Leftrightarrow H_{1}(\gamma)=\ldots=H_{r}(\gamma)=0\right)\right] .
$$

Since all algebraically closed fields of the same characteristic are elementarily equivalent [11, Theorem 1.13] it follows that $M$ can be replaced by any algebraically closed field $K$ of characteristic $p$. But this means that

$$
\mathcal{C}_{K}=\left\{\gamma \in \mathbf{A l g}_{K}(d): H_{1}(\gamma)=\ldots=H_{r}(\gamma)=0\right\},
$$

as required.

4.5. Proof of Theorem 2.4. The implication $(\mathrm{C}) \Rightarrow(\mathrm{A})$ has been proved in 3.6, $(\mathrm{B}) \Rightarrow(\mathrm{A})$ is obvious and the equivalence of $(\mathrm{C}),(\mathrm{D})$ and $(\mathrm{E})$ follows by standard model theory (see e.g. [11, Theorem 2.13], [2, Theorem 4.1.12]). 
Now Proposition 4.1 applied to $\mathcal{C}=\mathcal{W}$ proves $(\mathrm{A}) \Rightarrow(\mathrm{D})$ and finally it follows from Proposition 4.4 that (A) and (E) imply (B). The proof is complete.

REMARK 4.6. The author does not know if the condition: " $\mathcal{C}_{K}$ is closed in $\mathbf{A l g}_{K}(d)$ for every algebraically closed field and $\mathcal{C}$ is closed under elementary equivalence" implies that $\mathcal{C}$ is axiomatizable in $\operatorname{Alg}(d)$ (without fixing the characteristic).

5. Final comments. It seems interesting to consider classes of algebras inside which the wild ones form an axiomatizable class. If the whole class considered is axiomatizable we can use the above method in order to prove that the tame algebras induce an open subset of the set of algebras in the class. Below we analyse an example of this kind.

Let $\mathcal{Q} \subseteq \mathbf{A l g}(d)$ be the class of all basic quasitilted algebras [9] (of fixed dimension). Recall that $(K, R) \in \mathcal{Q}$ provided $R$ has global dimension less than or equal to 2 and every indecomposable finite-dimensional right $R$-module has either projective or injective dimension at most one. There is a criterion for tameness within this class of algebras. Using our notation we can formulate it in the following way: $(K, R) \in \mathcal{T}$ if and only if the Euler characteristic $\chi_{R}$ of $R$ is weakly nonnegative [20].

Recall that for a $K$-algebra $R$ of finite global dimension, $\chi_{R}$ is the quadratic form defined on the Grothendieck group $\mathbf{K}_{0}(R)$ of $R$ by the formula

$$
\chi_{R}([X])=\sum_{i=0}^{\infty}(-1)^{i} \operatorname{dim}_{K} \operatorname{Ext}_{R}^{i}(X, X)
$$

where $[X]$ denotes the class of a module $X$ in $\mathbf{K}_{0}(R)$. Let $e_{1}, \ldots, e_{n}$ be a complete set of primitive pairwise orthogonal idempotents of $R$. Given an $R$-module $X$ its dimension vector is defined to be

$$
\operatorname{dim}(X)=\left(\operatorname{dim}_{K} X e_{1}, \ldots, \operatorname{dim}_{K} X e_{n}\right) .
$$

There is an isomorphism $\mathbf{K}_{0}(R) \cong \mathbb{Z}^{n}$ given by $[X] \mapsto \operatorname{dim}(X)$. Let $C_{R}$ be the Cartan matrix of $R$, that is, $C_{R}=\left[c_{i j}\right]_{i, j=1, \ldots, n}$, where $c_{i j}=\operatorname{dim}_{K}\left(e_{j} R e_{i}\right)$ for $i, j=1, \ldots, n$. Then

$$
\chi_{R}(x)=x^{T}\left(C_{R}^{T}\right)^{-1} x
$$

for $x \in \mathbb{Z}^{n}$ (we identify $\mathbf{K}_{0}(R)$ with $\mathbb{Z}^{n}$ ). Here $A^{T}$ denotes the transpose of the matrix $A$. See [17] for details.

The form $\chi_{R}$ is said to be weakly nonnegative if $\chi_{R}(x) \geq 0$ for every vector $x$ with nonnegative coordinates.

Lemma 5.1. The class $\mathcal{Q}$ is axiomatizable and $\mathcal{Q} \cap \mathcal{T}$ is finitely axiomatizable as a subclass of $\mathcal{Q}$. 
Proof. $R$ being basic is equivalent to the fact that $r s-s r$ belongs to the Jacobson radical of $R$ for every $r, s \in R$ and this is easy to express as a first order sentence. The axiomatization of the homological dimension properties is standard, we refer to [11, Theorems $10.27,12.61]$.

In order to see that the class $\mathcal{Q} \cap \mathcal{T}$ is finitely axiomatizable as a subclass of $\mathcal{Q}$ we use the criterion from [20] mentioned above. It is enough to observe that there are only finitely many matrices which can be Cartan matrices of $d$-dimensional algebras. Moreover it is easy to write a first order sentence expressing that "the Cartan matrix of $R$ equals $C$ up to a simultaneous permutation of rows and columns" for every matrix $C$ with integral coefficients.

Using the arguments from Section 3 we get the following corollary.

Corollary 5.2. (a) There exists a number $N$ such that if $(K, R) \in$ $\mathcal{Q} \cap \mathcal{W}$ then there exists a $K\langle X, Y\rangle$-R-bimodule free of rank $m \leq N$ such that for any $m$-dimensional right $K\langle X, Y\rangle$-modules $U, V$ the $R$-modules $U \otimes_{K\langle X, Y\rangle} M$ and $V \otimes_{K\langle X, Y\rangle} M$ are isomorphic if and only if $U \cong V$.

Moreover, for every algebraically closed field $K$ :

(b) $\overline{(\mathcal{Q} \cap \mathcal{W})_{K}} \subseteq \mathcal{W}_{K}$.

(c) The set $(\mathcal{Q} \cap \mathcal{T})_{K}$ is open in $\mathcal{Q}_{K}$.

Assertions (b) and (c) seem to be easy to obtain by purely geometrical methods, but (a) does not seem to be obvious.

Let us note another consequence of axiomatizability of $\mathcal{T}$. Given a $K-$ algebra $R$ denote by $\tau_{R}$ the Auslander-Reiten transpose in $\bmod (R)$ [1]. By $[3$, Theorem $\mathrm{D}]$ if there is a number $m$ such that there are infinitely many pairwise nonisomorphic indecomposable modules $M$ of $K$-dimension $m$ such that $\tau_{R} M \not M$ then $R$ is of wild representation type.

Corollary 5.3. There is a function $\beta: \mathbb{N} \rightarrow \mathbb{N}$ such that for every $(K, R) \in \mathbf{A} \lg (d)$ : if there exist at least $\beta(m)$ pairwise nonisomorphic indecomposable modules $M$ of $K$-dimension $m$ such that $\tau_{R} M \neq M$ for some $m \in \mathbb{N}$ then $(K, R) \in \mathcal{W}$.

Proof. Let $\phi_{m, n}$ be a first order sentence expressing the property that there exists at least $n$ pairwise nonisomorphic indecomposable modules $M$ of $K$-dimension $m$ such that $\tau_{R} M \not M$. Then by the result of Crawley-Boevey $\left[3\right.$, Theorem D] mentioned above the set $\left\{\phi_{m, n}: n \in \mathbb{N}\right\}$ is inconsistent with the set of axioms for $\mathcal{T}$ for every $m$. By the Compactness Theorem (see e.g. $\left[15\right.$, Theorem 11.22]) there is a finite set $\left\{\phi_{m, 1}, \ldots, \phi_{m, \beta(m)}\right\}$ inconsistent with the axioms for $\mathcal{T}$. Therefore if $(K, R) \models \phi_{m, \beta(m)}$ for some $m$ then $(K, R) \in \mathcal{W}$. 
REMARK 5.4. (a) One can prove that $\phi_{m, \beta(m)}$ implies the existence of a bimodule $M$ as in Corollary 5.2(a) whose rank is bounded by a number depending only on $m$.

(b) Assume that we have an axiomatizable class $\mathcal{B}$ of algebras for which a converse of the result of Crawley-Boevey [3, Theorem D] is true in the following strong sense: for every $d$-dimensional wild algebra $R$ in $\mathcal{B}$ there exist infinitely many pairwise nonisomorphic indecomposable modules $M$ with dimension bounded by a number depending only on $d$ such that $\tau_{R} M \neq M$. Then Corollary 5.3 implies that the wild algebras form an axiomatizable subclass of $\mathcal{B}$.

Acknowledgements. The author thanks Professors S. Balcerzyk and J. Słomiński for indicating the reference [14].

\section{References}

[1] M. Auslander, I. Reiten and S. Smalø, Representation Theory of Artin Algebras, Cambridge Stud. Adv. Math. 36, Cambridge Univ. Press, 1995.

[2] C. C. Chang and H. J. Keisler, Model Theory, Stud. Logic Found. Math. 73, NorthHolland, 1973.

[3] W. W. Crawley-Boevey, On tame algebras and bocses, Proc. London Math. Soc. 56 (1988), 451-483.

[4] Yu. A. Drozd, Matrix problems and categories of matrices, Zap. Nauchn. Sem. Leningrad. Otdel. Mat. Inst. Steklov. 28 (1972), 144-153.

[5] - , Tame and wild matrix problems, in: Representations and Quadratic Forms, Akad. Nauk Ukrain. SSR, Inst. Mat., Kiev, 1979, 39-74.

[6] P. Gabriel, Finite representation type is open, in: Representations of Algebras, Lecture Notes in Math. 488, Springer, Berlin, 1975, 132-155.

[7] P. Gabriel, L. A. Nazarova, A. V. Roŭter, V. V. Sergĕ̌chuk and D. Vossieck, Tame and wild subspace problems, Ukrain. Math. J. 45 (1993), 335-372.

[8] C. Geiss, On degenerations of tame and wild algebras, Arch. Math. (Basel) 64 (1995), $11-16$.

[9] D. Happel, I. Reiten and S. Smalø, Tilting in abelian categories and quasitilted algebras, Mem. Amer. Math. Soc. 575 (1996).

[10] C. Jensen and H. Lenzing, Homological dimension and representation type of algebras under base field extension, Manuscripta Math. 39 (1982), 1-13.

[11] —, - Model Theoretic Algebra: with particular emphasis on fields, rings, modules, Algebra Logic Appl. 2, Gordon \& Breach, New York, 1989.

[12] S. Kasjan, Base field extensions and generic modules over finite dimensional algebras, Arch. Math. (Basel) 77 (2001), 155-162.

[13] H. Kraft, Geometric methods in representation theory, in: Representations of Algebras (Puebla, 1980), Lecture Notes in Math. 944, Springer, 1982, 180-258.

[14] J. Łoś, Quelques remarques, théorèmes et problèmes sur les classes définissables d'algèbres, in: Mathematical Interpretation of Formal Systems, North-Holland, 1955, 98-113.

[15] J. D. Monk, Mathematical Logic, Grad. Texts in Math. 37, Springer, New York, 1976. 
[16] J. A. de la Peña, On the dimension of module varieties of tame and wild algebras, Comm. Algebra 19 (1991), 1795-1807.

[17] C. M. Ringel, Tame Algebras and Integral Quadratic Forms, Lecture Notes in Math. 1099, Springer, 1984.

[18] I. R. Shafarevich, Basic Algebraic Geometry, Grundlehren Math. Wiss. 213, Springer, 1977.

[19] D. Simson, Linear Representations of Partially Ordered Sets and Vector Space Categories, Algebra Logic Appl. 4, Gordon \& Breach, 1992.

[20] A. Skowroński, Tame quasi-tilted algebras, J. Algebra 203 (1998), 470-490.

Faculty of Mathematics and Computer Science

Nicholas Copernicus University

Chopina 12/18

87-100 Torun, Poland

E-mail: skasjan@mat.uni.torun.pl

Received 18 October 2000;

in revised form 12 April 2001 